\title{
MUSCLE DISEASES
}

\section{UPDATE AND REVIEW OF CONGENITAL MYOPATHIES}

Congenital myopathies are reviewed by neuropathology researchers in New Delhi, India, and Mainz, Germany. The term 'congenital myopathy' (CM) was introduced with the discovery of 'central core disease,' a non-progressive myopathy described by Shy and Magee (1956). Molecular genetics, enzyme and immunohistochemical tests and electron microscopy have led to a better understanding of CM and their classification. CM is either structured or unstructured, with or without structural changes. Structured CM include, central core disease (autosomal dominant, mildly progressive or static; or autosomal recessive, more severe with onset in the first decade); multi-minicore disease (proximal muscle weakness, spinal rigidity, scoliosis, respiratory impairment, and external ophthalmoplegia); myotubular myopathies (type 1 fiber atrophy and central nuclei); X-linked myotubular myopathy (rapidly fatal in newborn boys, presenting with hypotonia and respiratory insufficiency, and arthrogryposis multiplex); centronuclear myopathy (autosomal dominant or recessive, or sporadic, neonatal and childhood forms, mildly progressive, central nuclei, type 1 predominance); nemaline myopathy (thread like rod inclusions on Gomori trichrome stains, 6 different forms, congenital to adult); actin aggregate myopathy (similar to nemaline myopathy, early onset, rapid course, rarely benign); desminopathy (slowly progressive, second to fourth decade distal weakness onset, cardiomyopathy, autosomal dominant or recessive); $a-B-$ crystallinopathy (similar to desminopathies, a myofibrillary myopathy); hyaline body myopathy (subsarcolemmal hyalinized bodies, rich in myofibrillary ATPase and myosin).

Unstructured CM: congenital fiber type disproportion (non-progressive childhood CM with relatively good prognosis, type 1 fiber predominance).

No clinical symptomatology is specific for an individual CM, but some clinical findings are more frequent in certain CMs; eg. ptosis or extraocular muscle weakness in multicore disease, nemaline myopathy, centronuclear myopathy, and congenital fiber type disproportion; scoliosis in desminopathies, and central core disease. (Sharma MC, Jain D, Sarkar C, Goebel HH. Congenital myopathies - a comprehensive update of recent advancements. Acta Neurol Scand May 009;119:281-292). (Respond: Dr MC Sharma, Department of Pathology, All India Institute of Medical Sciences, New Delhi-110029, India. E-mail: sharmamehar@yahoo.co.in).

COMMENT. An expertise in enzyme and immunohistochemical methods, electron microscopy, and molecular genetics is required to distinguish the rapidly expanding differential diagnosis of congenital myopathies. Clinical symptomatology is largely nonspecific.

\section{BENIGN RECURRENT SIXTH CRANIAL NERVE PALSIES}

A retrospective chart review of a cohort of 253 pediatric patients with sixth nerve palsies uncovered 30 cases of benign sixth nerve palsy, of which 9 were recurrent, in a study at University of Pennsylvania School of Medicine, Philadelphia. Sixth nerve palsy occurred alone in 225 patients, and the etiologies were as follows: $90(40 \%)$ had neoplasms, 25 
(11.1\%) were ascribed to increased intracranial pressure, $23(10.2 \%)$ to trauma, $14(6.2 \%)$ an infectious etiology, $10(4.4 \%)$ to vascular disease, $9(4 \%)$ inflammatory disorders, $6(2.7 \%)$ were congenital, $2(0.9 \%)$ secondary to surgery unrelated to neoplasm, and $1(0.4 \%)$ to radiation necrosis.

Benign sixth nerve palsy and recurrent cases had the following characteristics: a) isolated unilateral abduction palsy; b) without ptosis, papilledema or other neurologic signs; c) normal brain MRI; d) spontaneous improvement; e) without infection, inflammatory or other disease identified. The mean age at evaluation of the $30(13.3 \%)$ benign cases was 3 years. MRIs performed in 28 (93\%), and lumbar punctures in 6 were normal, Acetylcholine receptor antibody testing was negative in 11 (37\%), and lyme antibody titres, and anti-Gq1b antibody testing were normal. Only one child had some residual abduction deficit at 3-year follow-up. Four patients, including 3 with recurrences, had residual esotropia but full ductions, and all were referred for strabismus surgery. (Mahoney NR, Liu GT. Benign recurrent sixth (abducens) nerve palsies in children. Arch Dis Child May 2009;94:394-396). (Respond: Dr Nicholas R Mahoney, Scheie Eye Institute, 51 North $39^{\text {th }}$ Street, Philadelphia, PA 19104. E-mail: nicholas.mahoney@uphs.upenn.edu).

COMMENT. Proposed etiologies for benign sixth nerve palsies include ophthalmoplegic migraine, myasthenia gravis, and inflammation secondary to viral infections or vaccination (Lee MS, 1999). Age and gender are important, the age being younger in the recurrent cases, and girls are affected more frequently than boys. Cases related to vaccination are also prone to recurrence. (Yousuf et al, 2007). The cases in the above study were idiopathic.

\section{METABOLIC DISORDERS}

\section{VOLTAGE SENSORS IN HYPOKALEMIC PERIODIC PARALYSIS}

Researchers at the National Hospital, Queen Square, London, UK, conducted automated DNA sequencing of the S4 regions of CACNAIS and SCN4A in 83 patients with hypokalemic periodic paralysis (HypoPP). CACNA1S mutations were identified in 64 cases, and SCN4A or other CACNA1S mutations in 10, including 4 with new mutations. All mutations neutralized arginine residues in S4 segments. The patients with new mutations had the typical HypoPP phenotype: onset of attacks of muscle paralysis in first or second decade, at night or early morning, and low serum potassium. The findings were consistent with the gating pore cation leak hypothesis of HypoPP, and arginine mutations in S4 segments are involved in $90 \%$ cases. (Matthews E, Labrum R, Sweeny MG, et al. Voltage sensor charge loss accounts for most cases of hypokalemic periodic paralysis. Neurology May 5, 2009;72:1544-1547). (Response and reprints: Prof MG Hanna, Institute of Neurology and National Hospital for Neurology and Neurosurgery, Queen Square, London, WC1N 3BG, UK. E-mail: m.hanna@ion.ucl.ac.uk).

COMMENT. In an editorial, Cannon SC proposes that the remaining 10\% of HypoPP families with no identified mutation will also prove to be channelopathies, from a new class of molecular defect or different channel. (Neurology 2009;72:1540-1541). 